\title{
Long-Term Neuroprotective Effects of Hypothermia on Neonatal Hypoxic-Ischemic Brain Injury in Rats, Assessed by Auditory Brainstem Response
}

\author{
TAKUJI TOMIMATSU, HIROTSUGU FUKUDA, MASAYUKI ENDOH, JUNWU MU, \\ TAKESHI KANAGAWA, TAKAYOSHI HOSONO, TORU KANZAKI, KATSUMI DOI, \\ TAKESHI KUBO, AND YUJI MURATA
}

\author{
Departments of Obstetrics and Gynecology [T.T., H.F., M.E., J.M., T. Kanagawa, T.H., T. Kanzaki, Y.M.] \\ and Otolaryngology and Sensory Surgery [K.D., T. Kubo], Osaka University Faculty of Medicine, 2-2, \\ Yamada-oka, Suita, Osaka, 565-0871, Japan
}

\begin{abstract}
ABSTR
A method to assess long-term neurofunctional outcome of
hypothermia on immature brains has not yet been clearly estab-
lished. To investigate the effects of hypothermia on long-term
neurofunctional outcome, we studied brainstem function using
auditory brainstem response in adult rats after neonatal hypoxic-
ischemic brain injury. Seven-day-old rats underwent a combina-
tion of left common carotid artery ligation and subsequent ex-
posure to $8 \% \mathrm{O}_{2}$ for $1 \mathrm{~h}(n=17)$. The rats were divided into
three groups: hypothermia group $(n=6)$, normothermia group $(n$
$=6)$, and sham control group $(n=5)$. During recovery from the
hypoxic-ischemic insult, body temperature was reduced to $30^{\circ} \mathrm{C}$
for $24 \mathrm{~h}$ in the hypothermia group, but was kept at $37^{\circ} \mathrm{C}$ in the
normothermia and sham control group. Three months later the
rats were assessed by auditory brainstem response, then killed.
\end{abstract}
Perinatal hypoxic-ischemic brain injury is still one of the major causes of mortality and permanent neurologic disability. At present, there have not been any treatments that are proven to be effective, and the emphasis thus far has been on supportive therapy (1).

Although hypothermia has been reported to be the most effective method of neuroprotection in humans as well as in various animal models (2-4), long-term neuroprotective outcome of hypothermia has not yet been clearly established. Previous studies in immature animals have shown that postischemic hypothermia protects in the short-term (i.e. 1- to 7-d survival period) perspective (5-7), but there is no evidence with regard to long-term (i.e. 1- to 3-mo survival period)

Received February 14, 2002; accepted June 10, 2002

Correspondence: Takuji Tomimatsu, M.D., Department of Obstetrics and Gynecology, Osaka University Faculty of Medicine, 2-2, Yamada-oka, Suita, Osaka, 565-0871, Japan; e-mail: tomimatu@gyne.med.osaka-u.ac.jp

This work was supported by Grants-in-Aid for Scientific Research, No. 11307032 from the Ministry of Education, Japan.

DOI: 10.1203/01.PDR.0000042444.90269.2A
The normothermia group showed increased III-V latencies and wave $\mathrm{V}$ abnormalities. Hypothermia significantly ameliorated wave $\mathrm{V}$ abnormalities. Injury to the ipsilateral inferior colliculus was also reduced in the hypothermia group compared with that in the normothermia group, and the degree of damage assessed histologically correlated well with auditory brainstem response findings. The current study demonstrates that postischemic hypothermia may provide effective and long-lasting neurofunctional as well as histopathologic protection to the immature brain. (Pediatr Res 53: 57-61, 2003)
Abbreviation
ABR, auditory brainstem response

neurofunctional efficacy. At present, only one report has examined the effect of hypothermia on long-term neurofunctional outcome after neonatal cerebral hypoxia-ischemia, using some sensorimotor tests such as the postural reflex, but it failed to show long-term neurofunctional protection (8).

ABR is an early component of the auditory evoked potential, and provides objective information on the function of neural auditory pathways through the brainstem (9). Clinically, it has been widely used in evaluating brainstem function in perinatal asphyxiated infants $(10-12)$. Using the ABR, we have recently described brainstem dysfunction in adult rats exposed to hypoxia-ischemia in the neonatal period (13). In the present study, we examined whether hypothermia can improve functional outcome in the adult rat after neonatal cerebral hypoxiaischemia, using ABR.

\section{METHODS}

Subjects. This experiment was performed on 17 seven-dayold Sprague-Dawley rats obtained from S.L.C, Suita City, 
Japan. All experimental protocols were approved by the Osaka University Animal Care and Use Committee. The procedure for the hypoxic-ischemic injury was derived from the model developed by Rice et al. (14). The pups were anesthetized with halothane (induction, $4.0 \%$; maintenance, $2.0 \%$ ) in room air, then the left common carotid artery was surgically ligated with 5-0 surgical silk. After the procedure, the pups were returned to their dams and allowed to recover and to be fed for $1 \mathrm{~h}$. The pups were then placed in a chamber maintained in a water bath with the temperature kept at $37^{\circ} \mathrm{C}$. The chamber was perfused with a mixture of humidified $8.0 \%$ oxygen balanced with nitrogen for $1 \mathrm{~h}$. Then pups were divided into three groups, a hypothermia group $(n=6)$, a normothermia group $(n=6)$, and a sham control group $(n=5)$. Immediately after hypoxic exposure, the hypothermia group was placed in room temperature until the rectal temperature reached $30^{\circ} \mathrm{C}$, and then placed in a chamber in a water bath at a stable temperature of $30^{\circ} \mathrm{C}$ for $24 \mathrm{~h}$. The normothermia group remained in the chamber in the water bath at $37^{\circ} \mathrm{C}$ for $24 \mathrm{~h}$. The chamber was circulated with room air. The core temperature of the rat pups was measured by rectal probe. The sham control animals underwent only the same surgical procedure without arterial ligation or hypoxic exposure. After this, the animals were returned to the mother and reared normally. Animals were kept in a room (12 $\mathrm{h} / 12 \mathrm{~h}$ light/dark cycle) and weaned at $3 \mathrm{wk}$ of age.

$\boldsymbol{A B R}$. At 3 mo of age, ABR was recorded from the left side in response to click stimulations $(10 \mathrm{~Hz})$ from a speaker located $1 \mathrm{~cm}$ from one ear. Each animal was briefly anesthetized with ether, and s.c. needle electrodes were placed under the left external meatus (recording), vertex (reference), and under the right external meatus (ground). The stimulus intensity was a 136-dB sound pressure level. One thousand samples were averaged and filtered (50 to $6000 \mathrm{~Hz}$ ). Interpeak latencies were determined for waves I-III, I-V, and III-V. Amplitude of each wave was determined from the positive peak to the following trough, and the amplitude ratio of wave $\mathrm{V}$ to wave I was calculated in each group.

Tissue Preparation. For histologic analysis, the animals were deeply anesthetized with an overdose of sodium pentobarbital $(100 \mathrm{mg} / \mathrm{kg}$, i.p.) and transcardially perfused with $0.9 \%$ saline solution at $4{ }^{\circ} \mathrm{C}$, followed by $4 \%$ paraformaldehyde in $0.1 \mathrm{M}$ PBS, $\mathrm{pH} 7.4$ at $4^{\circ} \mathrm{C}$. Brains were removed and immersed in the same solution at $4^{\circ} \mathrm{C}$ for $24 \mathrm{~h}$, dehydrated with a graded series of ethanol and xylene, embedded in paraffin, and cut into 5- $\mu \mathrm{m}$-thick coronal sections at the level of the midbrain. Sections were deparaffinized in xylene and rehydrated in graded ethanol concentrations before staining. Slidemounted sections were stained with hematoxylin and eosin for viewing. Sections were analyzed with a Macintosh computer using the NIH Image program, version 1.62 , by a research assistant unaware of the treatment groups. With the aid of a microscope, the areas of the inferior colliculus in the ipsilateral and contralateral brainstem were determined. The area ratio of the inferior colliculus was calculated as follows:

Area ratio $=$ area of ipsilateral inferior colliculus / area of contralateral inferior colliculus

Statistical Analysis. Means were computed for each group of animals and analyzed using one-way ANOVA with post hoc
Fisher's protected least significant difference test. Significance was accepted at $p<0.05$ level.

\section{RESULTS}

As we reported previously $(15,16)$, the rectal temperature remained rather constant and stayed at approximately $0.5^{\circ} \mathrm{C}$ above the water bath temperature during the experimental procedure (data not shown).

A typical ABR pattern from 3-mo-old sham control and normothermic hypoxia-ischemia rats consisted of five waves within the first $5 \mathrm{~ms}$ after click stimulation (Fig. 1). In the normothermic hypoxia-ischemia rat, there were abnormalities in both a prolongation of wave $\mathrm{V}$ latency and a decreased amplitude of wave $\mathrm{V}$ compared with the sham control rat. There were no significant differences for wave I latencies. Figure 2 shows the mean interpeak latency differences for group comparisons for waves I-III and III-V. Analysis of the interpeak latency differences did not detect significant group differences for wave I-III interpeak latencies (Fig. 2A). On the contrary, wave III-V interpeak latency was significantly longer in the normothermia group than in the sham control group $(p<$ 0.05 ; Fig. 2B). Although there was a clear tendency for the hypothermia group to show a shorter interpeak latency, wave III-V interpeak latency between the normothermia group and the hypothermia group did not significantly differ. However, the amplitude ratio of wave $\mathrm{V}$ to wave I was significantly lower in the normothermia group than in either the sham control group or in the hypothermia group ( $p<0.05$; Fig. 3 ).

Giving special emphasis to the inferior colliculus, which usually receives widespread input from other structures of the auditory system (17), we histologically studied the midbrain after ABR recording. Figure 4 shows the effect of hypothermia after neonatal hypoxia-ischemia on midbrain damage in 3-moold rats. Figure $4 A$ shows a representative coronal brain section at the inferior colliculus level assessed 3 mo after neonatal hypoxia-ischemia. A mild atrophic appearance in the ipsilateral inferior colliculus was observed in the normothermia group (Fig. $4 A$ ). The injury to the inferior colliculus was assessed in each experimental group. The area ratio of the inferior colliculus was 0.834 in the normothermia group. Hypothermia significantly reduced this damage to 0.939 (Fig. $4 B$ ) at 3 mo of age.

To evaluate whether there was a correlation between the degree of midbrain damage and functional deficits, correlation coefficients were calculated between the area ratio of inferior colliculus and ABR findings. Figure 5 presents the correlation of the two ABR measurements to the area ratio of inferior colliculus as assessed histologically. All ABR measurements exhibited highly significant correlation coefficients.

\section{DISCUSSION}

In the present study, postischemic hypothermia provided effective and long-lasting neuroprotection to the immature auditory system. To our knowledge, this is the first study that proved persistent neuroprotective effects of the hypothermia up to adulthood, by demonstrating neurofunctional as well as histologic improvements from hypoxic-ischemic insults given at neonatal period. 
(A)

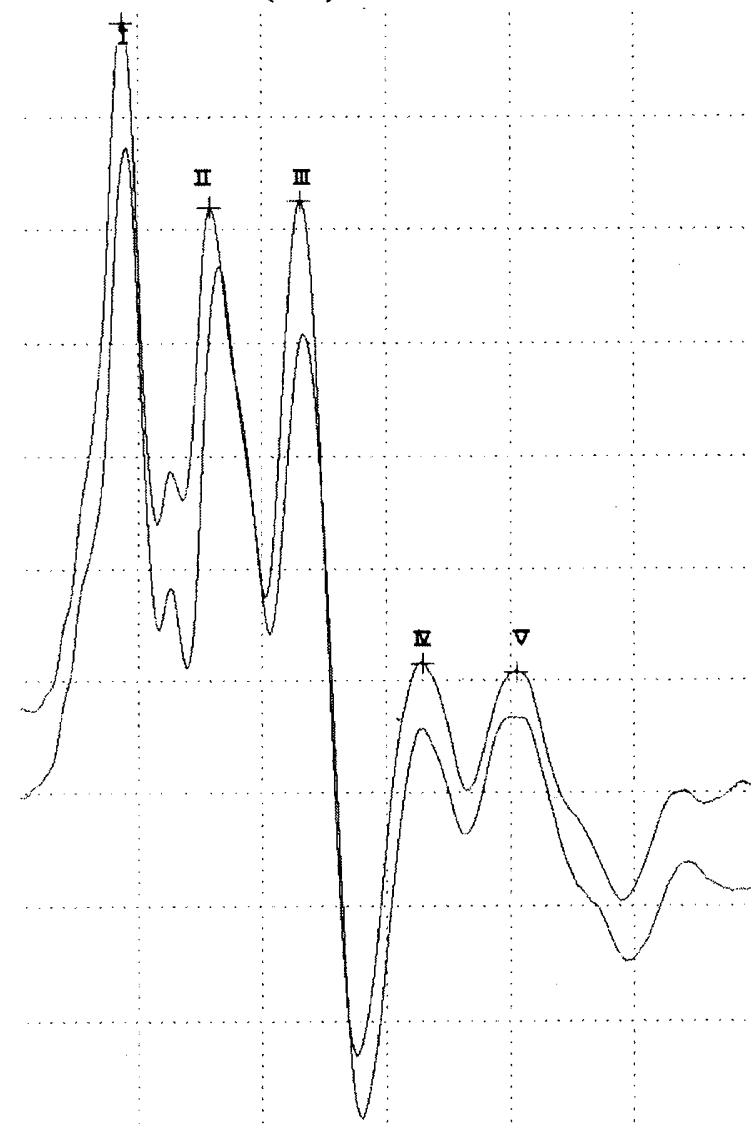

(B)

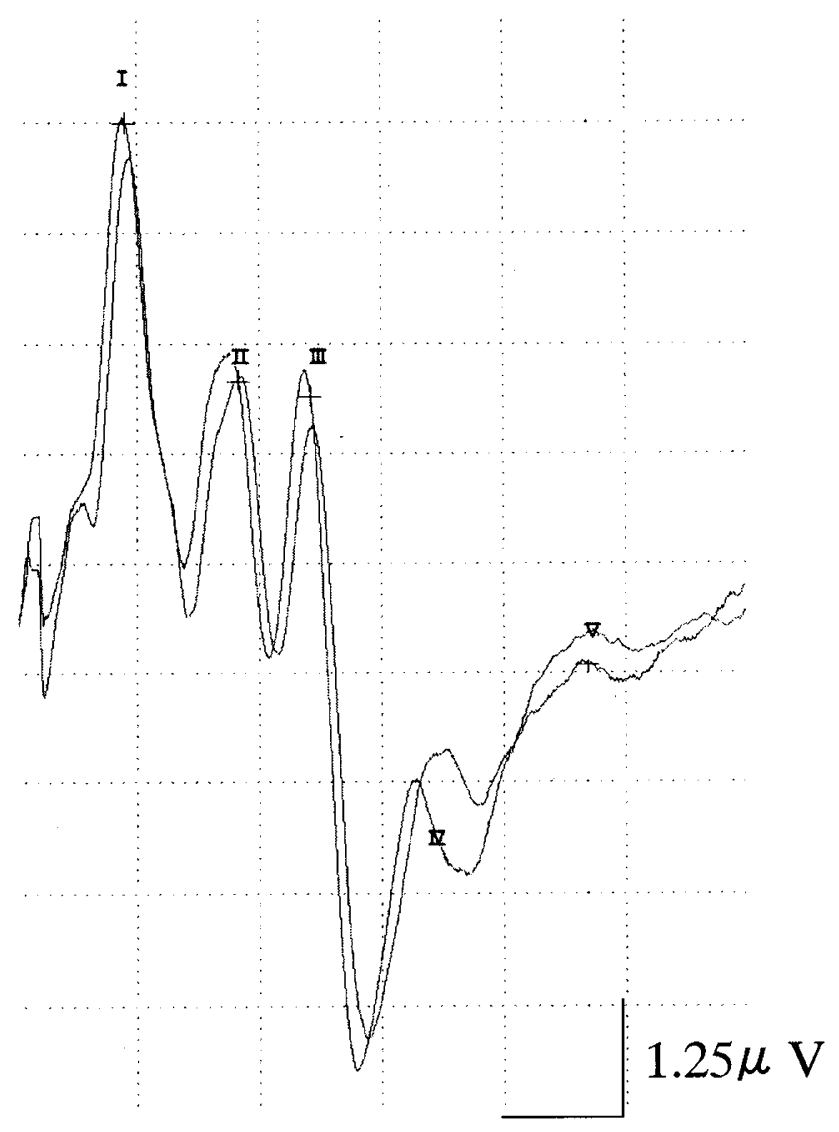

$1 \mathrm{msec}$

Figure 1. Typical ABR recordings in 3-mo-old sham control $(A)$ and normothermic hypoxia-ischemia $(B)$ rat. Note that a prolongation of wave $\mathrm{V}$ latency and decreased amplitude of wave $\mathrm{V}$ were seen in normothermic hypoxia-ischemia rat.

Since Busto et al. (18) first reported using adult gerbils, numerous studies have reported the neuroprotective effects of hypothermia in various animal hypoxic-ischemic models, including immature animals (19-21). However, these studies are limited by the use of short survival times, the lack of functional assessment, or both. Hypoxia-ischemia-induced cell death has been shown to continue for weeks or months, and recent studies have demonstrated that histologic preservation does not necessarily translate into preserved neuronal function $(22,23)$. Accordingly, there is a need for long-term neurofunctional assessment after postischemic hypothermia. Fortunately, recent studies using adult animals show that prolonged postischemic hypothermia provides long-lasting functional protection $(24,25)$. When considering perinatal asphyxia, the result obtained in adult animals cannot be directly transposed onto neonatal animals. Both the mechanism of injury and response to the therapeutic interventions are likely to be different in neonatal animals. For example, immature brains are thought to be more resistant to hypoxia-ischemia and show a faster evolution of the injury (26). Resistance of immature brain is thought to be achieved by mechanisms such as lower energy demand (27), the ability use lactate and ketone bodies for energy (28), and early induction of heat shock protein (29). It is therefore

\section{Interpeak Interval}

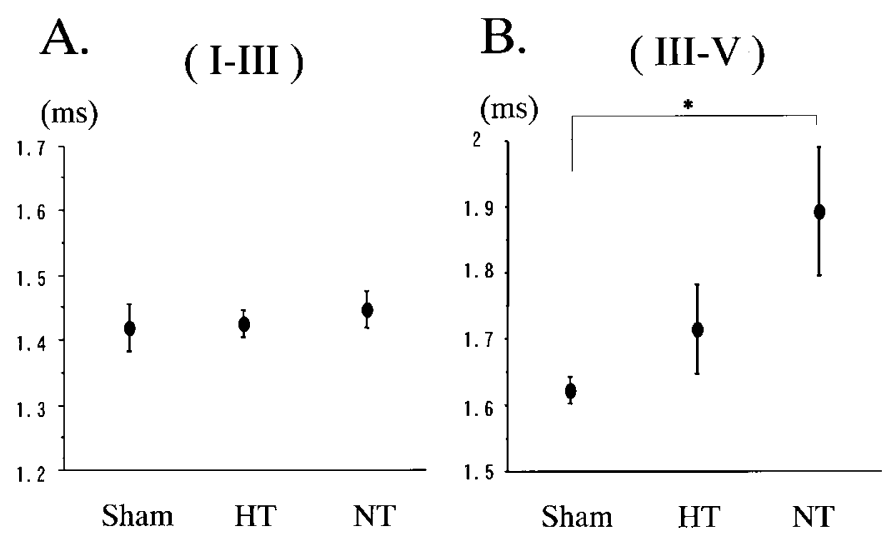

Figure 2. Mean interpeak latency differences for waves I-III and III-V. The interpeak latency differences were not significantly different for wave I-III interpeak latencies $(A)$. Latency of wave I was not significantly different. There were also no significant differences for wave I latencies. Wave III-V interpeak latency was significantly longer in normothermia group $(N T)$ than in sham control group (Sham; $\left.{ }^{*} p<0.05 ; B\right)$. Wave III-V interpeak latency between NT and hypothermia group $(H T)$ did not significantly differ. Values are means; error bar indicates 1 SEM. 
Amplitude Ratio of Wave V / Wave I

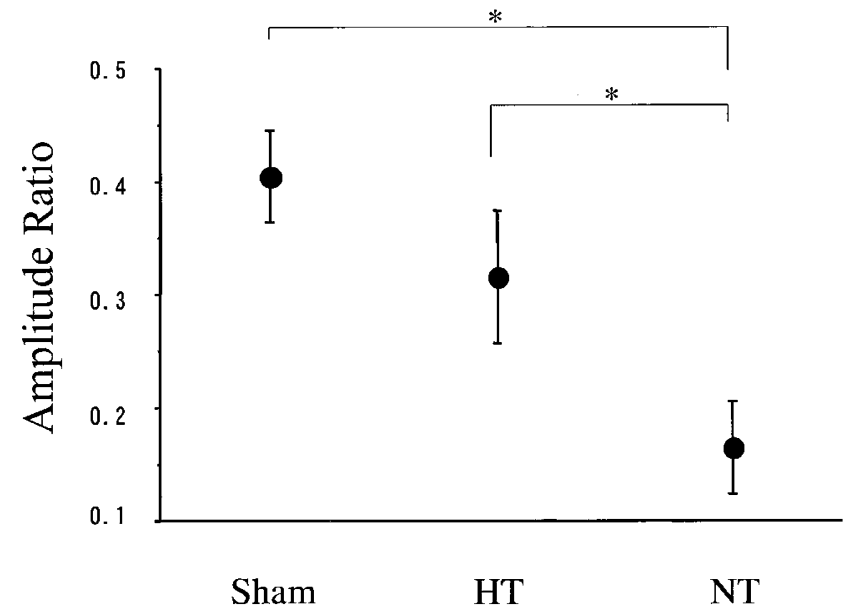

Figure 3. Amplitude ratio of wave V to wave I. Normothermia group (NT) was significantly lower than both sham control (Sham) and hypothermia (HT) groups $(* p<0.05)$. Values are means; error bar indicates 1 SEM.

essential that any therapeutic strategy intended for human infants should be thoroughly tested in newborn animal models.

Evoked ABR is an objective measure of CNS integrity (9). Clinically, the ABR has been widely used for evaluating peripheral auditory function and neural brainstem integrity in perinatal asphyxiated infants $(10-12)$. However, there have been surprisingly few studies in which ABR was applied to the assessment of long-term outcome in various animal hypoxicischemic models. One study reported an increased I-V interval and decreased wave $\mathrm{V}$ to wave I ratio in young rabbits subjected to hypoxia (30). However, the study was limited by the use of ABR immediately after hypoxic insult. In the present study, the ABR provides a stable and objective behavioral measure after neonatal cerebral hypoxia-ischemia. Moreover, the ABR measurements correlate to the extent of the injury, suggesting that this test is useful in evaluating treatment effects for a longer period of time. It is generally considered that the auditory nerves generate peak I, the cochlear nucleus, peak II, the superior olivary nucleus, peak III, the lateral lemniscus, peak IV, and the inferior colliculus, peak V in rat (31). Thus, increased III-V latencies and wave $\mathrm{V}$ abnormalities observed in the present study are probably attributed to generators lying primarily within the midbrain. The inferior colliculus receives widespread input from other structures of the auditory system (17). In the current study, hypothermia significantly reduced damages to the ipsilateral inferior colliculus in 3-mo-old neonatal hypoxia-ischemia rats. Although the basis for the change in electrophysiologic response in this model will remain uncertain until there is a more complete understanding of the brainstem auditory pathway, it is possible that the neuroprotective effects of hypothermia on the inferior colliculus may partly contribute to the preservation of ABR findings.

Indeed, it is generally thought the main lesions in this model involve the cortex, hippocampus, and striatum, but one report described reduced blood flow to the ipsilateral inferior colliculus in this neonatal hypoxic-ischemic model (32). We have

\section{(A) Representative coronal section at the midbrain level}

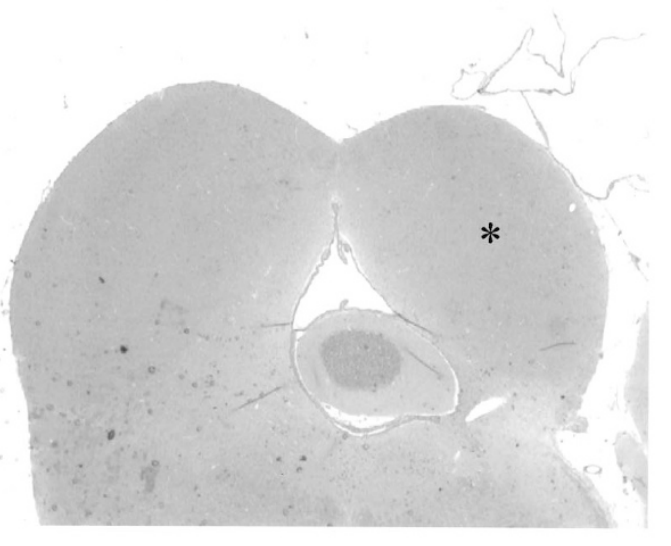

Normothermic H-I rat

(B) Area ratio of inferior colliculus

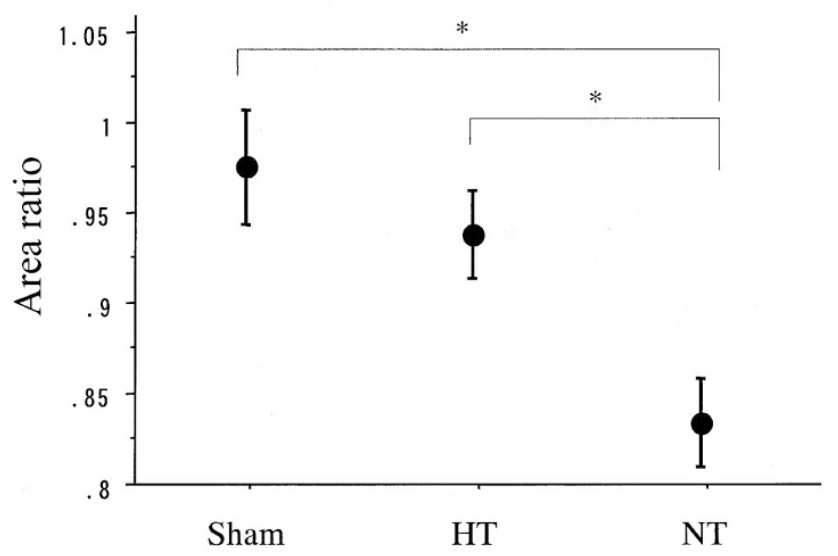

Figure 4. Effects of hypothermia after neonatal hypoxia-ischemia on brainstem damage in a 3-mo-old rat. $A$, representative coronal brain section of normothermic hypoxic-ischemic rat assessed 3 mo after left carotid ligation. A coronal section at the midbrain level showed a relatively mild atrophic appearance in the ipsilateral inferior colliculus (indicated by [squlo]). $B$, area ratio (area of ipsilateral inferior colliculus to area of contralateral inferior colliculus) in 3-mo-old rats $(n=5-6)$. Area ratio was significantly lower in normothermia group $(N T)$ than in either sham control (Sham) or hypothermia (HT) group $\left({ }^{*} p<0.05\right)$. Values are means; error bar indicates 1 SEM.

recently described an increased I-V interval in adult rats exposed to hypoxia-ischemia in the neonatal period, and the presence of activated caspase-3-positive cells in the ipsilateral inferior colliculus $24 \mathrm{~h}$ after neonatal hypoxia-ischemia, suggesting that the apoptosis signal pathway may be activated in this region (13). In addition to several neuroprotective mechanisms of hypothermia suggested in some studies $(5,33-35)$, we recently described that postischemic hypothermia blocks caspase-3 activation after neonatal cerebral hypoxia-ischemia (15). It is possible that hypothermia might suppress apoptotic cell death in this region, leading to the preservation of auditory function in the adult rat after neonatal cerebral hypoxia-ischemia. 
A

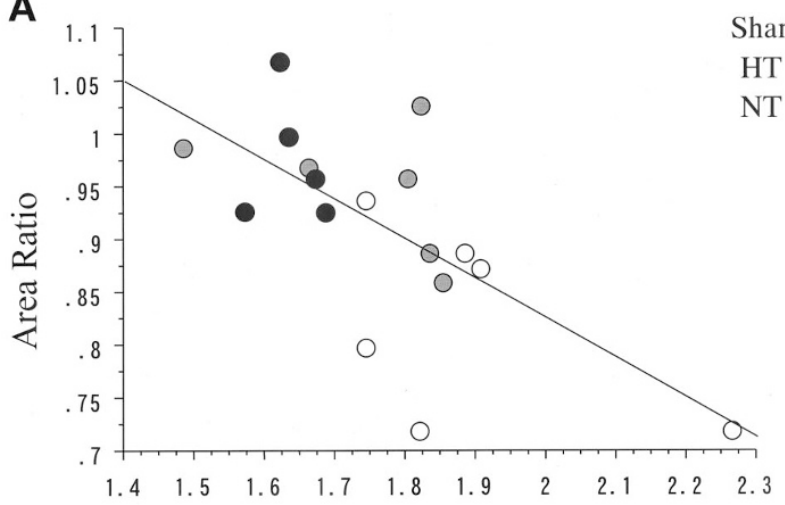

III-V interpeak latency

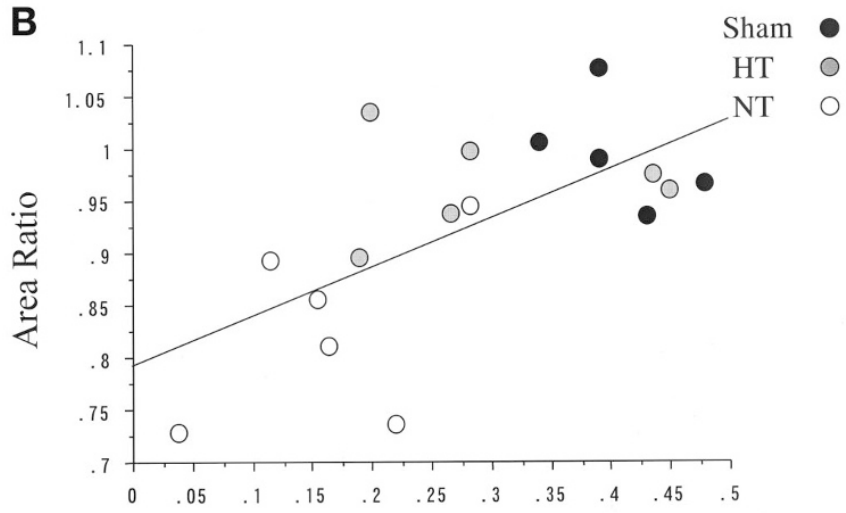

Amplitude ratio of wave $\mathrm{V} /$ wave I

Figure 5. III-V interpeak latency $(r=-0.626 ; p=0.0275 ; A)$ and amplitude of wave $\mathrm{V}$ to wave I $(r=0.620 ; p=0.0295 ; B)$ were plotted against area ratio (area of ipsilateral inferior colliculus to area of contralateral inferior colliculus) in sham control (Sham), hypothermia group (HT), and normothermia group (NT).

\section{CONCLUSIONS}

In summary, the current study demonstrated using ABR that the improvement of functional outcome by hypothermia can continue to adulthood in rats after neonatal cerebral hypoxiaischemia. Postischemic hypothermia may provide effective and long-lasting neuroprotection for the immature brain. Although a recent clinical study failed to show long-term efficacy of hypothermia, it supported the safety of hypothermia with no evidence of late adverse effects in any infants (36). Our data strongly warrant further clinical investigation of hypothermia in hypoxic-ischemic encephalopathy.

\section{REFERENCES}

1. Berger R, Garnier Y 1999 Pathophysiology of perinatal brain damage. Brain Res Rev 30:107-134

2. Colbourne F, Sutherland GR, Corbett D 1997 Postischemic hypothermia: a critical appraisal with implications for clinical treatment. Mol Neurobiol 14:171-201

3. Gunn AJ 2000 Cerebral hypothermia for prevention of brain injury following perinatal asphyxia. Curr Opin Pediatr 12:111-115

4. Nedelcu J, Klein MA, Aguzzi A, Martin E 2000 Resuscitative hypothermia protects the neonatal rat brain from hypoxic-ischemic injury. Brain Pathol 10:61-71

5. Edwards AD, Yue X, Squier MV, Thorensen M, Caby EB, Penrice J, Cooper CE, Wyatt JS, Reynolds EOR, Mehmet H 1995 Specific inhibition of apoptosis after cerebral hypoxia-ischemia by moderate post-insult hypothermia. Biochem Biophys Res Commun 217:1193-1199

6. Gunn AJ, Gunn TR, de Haan HH, Williams CE, Gluckman PD 1997 Dramatic neuronal rescue with prolonged selective head cooling after ischemia in fetal lambs. J Clin Invest 99:248-256

7. Thoresen M, Bagenholm R, Loberg EM, Apricenta F, Kjellmer I 1996 Posthypoxic cooling of neonatal rats provides protection against injury. Arch Dis Child 74:F3-F9

8. Bona E, Hagberg H, Loberg EM, Bagenholm R, Thoresen M 1998 Protective effects of moderate hypothermia after neonatal hypoxia-ischemia: short- and long-term outcome. Pediatr Res 43:738-745

9. Jacobson JT 1985 The Auditory Brainstem Response. College-Hill Press, San Diego, pp 3-12

10. Kileny P, Connelly C, Robertson C 1980 Auditory brainstem responses in perinatal asphyxia. Int J Pediatr Otorhinolaryngol 2:147-159

11. Hecox KE, Cone B 1981 Prognostic importance of brainstem auditory evoked responses after asphyxia. Neurology 31:1429-1433

12. Yasuhara A, Kinoshita Y, Hori A, Iwase S, Kobayashi Y 1986 Auditory brainstem response in neonates with asphyxia and intracranial haemorrhage. Eur J Pediatr 145:347-350

13. Tomimatsu T, Fukuda H, Endoh M, Mu J, Watanabe N, Kohzuki M, Fujii E, Kanzaki T, Oshima K, Doi K, Kubo T, Murata Y 2002 Effects of neonatal hypoxic-ischemic brain injury on skilled motor tasks and brainstem function in adult rats. Brain Res 926:108-117

14. Rice JE, Vannucci RC, Brierley JB 1981 The influence of immaturity on hypoxicischemic brain damage in the rat. Ann Neurol 9:131-141

15. Fukuda H, Tomimatsu T, Watanabe N, Mu J, Kohzuki M, Endoh M, Fujii E, Kanzaki T, Murata Y 2001 Post-ischemic hypothermia blocks caspase-3 activation in the newborn rat brain after hypoxia-ischemia. Brain Res 910:187-191

16. Tomimatsu T, Fukuda H, Endoh M, Watanabe N, Mu J, Kohzuki M, Fujii E, Kanzaki T, Murata Y 2001 Effects of hypothermia on neonatal hypoxic-ischemic brain injury in the rat: phosphorylation of Akt, activation of caspase-3-like protease. Neurosci Lett 312:21-24

17. Coleman JR, Clerici WJ 1987 Source of projections to subdivisions of the inferior colliculus in the rat. J Comp Neurol 62:215-226

18. Busto R, Dietrich WD, Globus MY, Valdes I, Scheinberg P, Ginsberg MD 1987 Small differences in intraischemic brain temperature critically determine the extent of ischemic neuronal injury. J Cereb Blood Flow Metab 7:729-738

19. Chen H, Chopp M, Zhang ZG, Gracia JH 1992 The effect of hypothermia on transient middle cerebral artery occlusion in the rat. J Cereb Blood Flow Metab 12:923-927

20. Yanamoto H, Hong SC, Soleau S, Kassell NF, Lee KS 1996 Mild postischemic hypothermia limits cerebral injury following transient focal ischemia in rat neocortex. Brain Res 718:207-211

21. Corbett D, Hamilton M, Colbourne F 2000 Persistent neuroprotection with prolonged postischemic hypothermia in adult rats subjected to transient middle cerebral artery occlusion. Exp Neurol 163:200-206

22. Van der Staay FJ, Augstein KH, Horvath E 1996 Sensorimotor impairments in rats with cerebral infarction, induced by unilateral occlusion of the left middle cerebral artery: strain differences and effects of the occlusion site. Brain Res 735:271-284

23. Corbett D, Nurse S 1998 The problem of assessing effective neuroprotection in experimental cerebral ischemia. Prog Neurobiol 54:531-548

24. Colbourne F, Corbett D, Zhao Z, Yang J, Buchan AM 2000 Prolonged but delayed postischemic hypothermia: a long-term outcome study in the rat middle cerebral artery occlusion model. J Cereb Blood Flow Metab 20:1702-1708

25. Yanamoto H, Nagata I, Niitsu Y, Zhang Z, Xue JH, Sakai N, Kikuchi H 2001 Prolonged mild hypothermia therapy protects the brain against permanent focal ischemia. Stroke 32:232-239

26. Farmer SF, Harrison LM, Ingram DA, Stephans JA 1991 Plasticity of central motor pathways in children with hemiplegic cerebral palsy. Neurology 41:1505-1510

27. Duffy TE, Kohle SJ, Vannucci RC 1975 Carbohydrate and energy metabolism in perinatal rat brain: relation to survival in anoxia. J Neurochem 24:271-276

28. Young RS, Petroff OA, Chen B 1991 Preferential utilization by isolated cells from early neonatal rat brain. J Neurochem 57:1700-1707

29. Xia XY, Ikeda T, Ota A, Xia YX, Sameshima H, Ikenoue T, Toshimori K 1999 Heat shock protein 72 expression and microtubule-associated-protein 2 disappeared after hypoxia-ischemia in the developing brain. Am J Obstet Gynecol 180:1254-1262

30. Inagaki M, Kaga M, Isumi H, Hirano S, Takashima S, Nanba E 1997 Hypoxiainduced ABR change and heat shock protein expression in the pontine auditory pathway of young rabbits. Brain Res 757:111-118

31. Funai H, Funasaka S 1983 Experimental study on the effect of inferior colliculus lesions upon auditory brainstem response. Audiology 22:9-19

32. Vannucci RC, Lyons DT, Vasta F 1988 Regional cerebral blood flow during hypoxia-ischemia in immature rats. Stroke 19:245-250

33. Kader A, Frazzini VJ, Baker CJ, Solomon RA, Trifiletti RR 1994 Effect of mild hypothermia on nitric oxide synthesis during focal cerebral ischemia. Neurosurgery 35:272-277

34. Busto R, Globus MYT, Dietrich WD, Martinez E, Valdes I, Ginsberg MD 1989 Effect of mild hypothermia on ischemia-induced release of neurotransmitters and free fatty acids in rat brain. Stroke 20:904-910

35. Kil HY, Zhang J, Piantadosi CA 1996 Brain temperature alters hydroxyl radical production during cerebral ischemia/reperfusion in rats. J Cereb Blood Flow Metab 16:100-106

36. Battin MR, Dezoete JA, Gunn TR, Gluckman PD, Gunn AJ 2001 Neurodevelopmental outcome of infants treated with head cooling and mild hypothermia after perinatal asphyxia. Pediatrics 107:480-484 